\title{
Effect of Sappan Wood Ethanol Extracts (Caesalpinia sappan. $L$ ) to the Sperm Motility, Viability, and Concentration of Male Wistar Rats
}

\author{
Nadiyah, ${ }^{1}$ Andri Rezano, ${ }^{2}$ Sunarjati Sudigdoadi ${ }^{3}$ \\ ${ }^{1}$ Faculty of Medicine Universitas Padjadjaran, ${ }^{2}$ Department of Anatomy, Cell Biology and \\ Physiology Faculty of Medicine, Universitas Padjadjaran, ${ }^{3}$ Department of Microbiology and \\ Parasitology Faculty of Medicine Universitas Padjadjaran
}

\begin{abstract}
Background: Idiopathic male infertility becomes an issue because of the limited management options and increasing number of infertile couples. Sappan wood (Caesalpinia sappan. $L$ ) is an herbal plant predicted to have fertility and spermatogenesis enhancing effects through antioxidant properties. This study aimed to observe the effects of sappan wood ethanol extract in improving sperm quality of male wistar rat based on sperm motility, viability, and concentration.
\end{abstract}

Methods: This was an experimental laboratory study by using sappan wood ethanol extracts as material and male wistar rat as the experimental animal. Twenty-four adult male wistar rats were divided into 4 groups including rats which were treated with aquades, 30,60, and $120 \mathrm{mg} / \mathrm{kg} / \mathrm{day} / \mathrm{orally}$ of ethanol extract of sappan wood, respectively, during 35 days. After this period, the animals were sacrificed. Then the epididymis were taken for samples to calculate sperm motility percentage, sperm viability percentage and sperm concentration. Comparison between control group and the treated group was analyzed by one-way Annova.

Results: There were significant differences between the control group and the group with $120 \mathrm{mg} / \mathrm{kg}$ of sappan wood ethanol extracts in the percentage of motility ( $36.39 \%$ and $51.75 \%$; p-value 0.001 ), viability percentage (55.36\% and $66.63 \%$, p-value 0.031$)$, and sperm concentration $(54.47 \mathrm{million} / \mathrm{ml}$ and 47.79 million/ml; p-value 0.001).

Conclusions: The ethanol extract of sappan wood with a dose of $120 \mathrm{mg} / \mathrm{kg}$ for 35 days is able to improve sperm motility percentage, sperm viability percentage, and sperm concentration of male wistar rat.

Keywords: Antioxidant, Caesalpinia sappan. L, infertility

\section{Introduction}

Infertility is a global health problem affect $15 \%$ couples worldwide. ${ }^{1}$ Approximately 50 million couples suffered from infertility by year 2010 compared to 42 million couples suffered from infertility by year 1990. The highest prevalence of infertility occurs in South Asia, Sub-Saharan Africa, North Africa, Middle East, Central Europe, Eastern Europe, and Central Asia. ${ }^{2}$ Infertility has become an issue for its impact to the quality of life of married couples. Infertile couples have had to undergo a lengthy treatment and management regimen that may place burdens on their life. The lengthy treatment has affected psychological problems of infertile couple and also there were embarrassment and social stigma felt by

\section{childless couple. ${ }^{3}$}

Infertility can be found in either males or females. Approximately $40 \%$ of infertility cases are caused by male infertility. In male infertility cases, approximately 30 to $45 \%$ of the cases have unknown causes or is defined as idiopathic male infertility. ${ }^{1}$ The etiology and pathophysiology of idiopathic male infertility are still unknown. The existing treatment and management for idiopathic male infertility are still very limited and lacking in efficacy. ${ }^{4}$

Empirical therapies to treat idiopathic male infertility are antioxidant supplements. Several studies stated that combination of antioxidant supplements, such as vitamin C, vitamin E, zinc, selenium, L-carnitine, and coenzyme Q10, taken orally may increase sperm quality based on sperm concentration, amount, and motility.

Correspondence: Nadiyah, Faculty of Medicine, Universitas Padjadjaran, Jalan Raya Bandung-Sumedang Km.21, Jatinangor, Sumedang, Indonesia, Phone: +62 85782318569 Email: Nadiyah.96.28@gmail.com 
Antioxidants may increase sperm quality by preventing sperm damage caused by oxidative stress that may occur in male infertility. ${ }^{5}$

Sappan wood (Caesalpnia sappan. L) is a species of flowering tree in the legume family, Fabaceae, that is native to Southeast Asia and commonly found in Indonesia. The main component of sappan wood that act as an antioxidant is brazilin. The structure of hydroxyl chain in brazilin contains phenolic compound that inhibit free radicals or reactive oxygen species (ROS) production. ${ }^{6}$ Regulation of ROS plays an important part in normal spermatogenesis and sperm production. ${ }^{7}$ Therefore, sappan wood may affect in improving sperm quality. This study aimed to observe the effects of sappan wood ethanol extract on sperm quality parameters based on sperm motility percentage, sperm viability percentage, and sperm concentration of male wistar rat.

\section{Methods}

This was an experimental study which conducted in Laboratory of Natural Products, Faculty of Pharmacy, Universitas Padjadjaran for the extraction of the Sappan Wood; Laboratory of Pharmacology and Therapeutics, Faculty of Medicine, Universitas Padjadjaran for animal experiments, and the Laboratory of Anatomy and Cell Biology, Faculty of Medicine, Universitas Padjadjaran for the semen analysis in male rat, from September to December 2015. This study was approved by the Health Research Ethics Committee, Faculty of Medicine, Universitas Padjadjaran. The object of the study were male wistar rats.

Sappan wood used in the study was obtained from the Research Institute of Medicinal and Aromatic Plants, Bogor and was determined in Herbarium Bogoriense Institute, Lembaga Ilmu Pengetahuan Indonesia (LIPI). Sappan wood ethanol extract was processed in Laboratory of Nature Materials, Faculty of Pharmacy, Universitas Padjadjaran. The extraction process started with pulverizing the sappan wood. Shaved sappan wood firstly washed and thinly sliced, and then dried at room temperature until the water content reduce. The extract was processed by maceration technics, using ethanol $70 \%$ for 24 hours in macerator. Macerate was separated by filtration using a Buchner funnel which connected to a vacuum. The filtering process was needed to be completed at least twice by using the same type and amount of solvent. Then, all macerate was collected and evaporated using a rotary evaporator to obtain a yellow-brown viscous extract. ${ }^{8}$ Sappan wood ethanol extract formed into suspension with $100 \mathrm{ml}$ of sodium carboxyl methyl cellulose (CMC Na) 0.5\%.

By using the Frederer's formula, the total sampel obtained were 24 rats. Twenty-four male wistar rats (Rattus norvegicus) were treated for 3 dose variation of sappan wood. Rats were obtained from the Inter-University Laboratory of Biological Sciences Institute of Technology Bandung with average age and weight 2-3 months old and 180-200 gr, respectively. Rats were acclimatized beforehand with the atmosphere of a research laboratory for 7 days. The inclusion criterion were all rats which had constant body weight between 180-200 grams during the adaptation period. Exclusion criterion were rats which had weight loss $>10 \%$ during the adaptation period. The maintenance was done under lighting process contained 12 hours of darkness and 12 hours of light with room temperature $26^{\circ} \mathrm{C}$ and humidity of $60 \%$. Diet for rats was in the form of small pellets and water administered daily and ad libitum to all rat.

This study divided the objects into 4 groups, 1) The control group $(n=6)$, only given aquades, 2) Group I, given a suspension of sappan wood ethanol extract $30 \mathrm{mg} / \mathrm{kg}$ body weight/day of dose, orally, 3) Group II, given a suspension of the sappan wood ethanol extract with $60 \mathrm{mg} / \mathrm{kg}$ body weight/ day of dose, orally, and 4) Group III, given suspension of sappan wood ethanol extract with $120 \mathrm{mg} / \mathrm{kg}$ body weight/day of dose, orally. The Sappan wood ethanol extract was given for 35 days by oral gavage.

On the 36th day, the rat was anaesthetized using ketamine (70 mg/body weight) and xylazine (10 mg/body weight) and then killed by cervical dislocation. The posterior part of the dextra and sinistra distal cauda epididymis, to the ends of the ductus deferens were isolated and placed in $0,25 \mathrm{ml} \mathrm{NaCl} 0.9 \%$ in petri dish. Sperm released by clamping the end of the epididymis and then pressed in the same direction and clamping the other end of the epididymis and also pressed in the same direction. The process was carried out several times and then stirred until homogeneous suspension obtained. Calculation of sperm motility percentage, sperm viability percentage and amount or concentration of sperm was done afterwards.

The percentage of sperm motility and sperm viability were calculated by evaluating 
the minimum of 200 sperm in 5 visual fields under a light microscope with a magnification of 400 times. Sperm were then classified based on the type of movement, progressive moving sperm, non-progressive, and not-moving sperm (immotile). The viability percentage was calculated by evaluating the viability of 200 sperm by staining using eosin-Y $0.5 \%$ under a microscope with a magnification of 400 times. Viable sperm categorized as sperm which had white or pink head color while unviable sperm had red or dark pink of head color. The calculation of the sperm concentration was done by using the counting chamber (improved Neubauer hemocytometer) which was calculated on the central grid. ${ }^{9}$

Comparison of the values of control and treated groups was carried out by statistical analysis of variance (ANOVA), followed by Tukey's test. This study used a confidence interval of $95 \%$ and the result stated to be statistically significant if the $p$-value $<0.05$. The normality of obtained data was tested using the Shapiro-Wilk test, due to the total number sample was $\leq 50$ and data homogeneity test was using Levene test. If the data were not normally distributed and the variance of the data was not homogeneous, then Kruskal Wallis non parametric test was performed. If the result was significant, post hoc test would be performed later.

\section{Results}

The results of sperm motility percentage, sperm viability percentage and sperm concentration calculation were shown in Figure 1 and Figure 2.

ANOVA test results on motile sperm percentage showed a significant difference between the treatment groups with $p$-value 0.005 (Table 1). Post hoc test was conducted to determine which group had significant percentage motility. Post hoc results showed there were significant differences between the control group and group III ( $\mathrm{p}$ value 0.001 ), between group I and group III ( $p$ value 0.011 ), and between group II and group III ( $p$ value 0.005).

The ANOVA test results on viable sperm percentage showed significant differences between three groups; with p-value 0.002 (Table 1). Post hoc test showed significant difference between the control group with group III ( $p$ value 0.031 ), between group I and group II ( $p$ value 0.001 ), and between group I and group III ( $\mathrm{p}$ value 0.001 ).

ANOVA test results on the amount/ concentration of sperm showed a significant difference between the three groups with p-value 0.001 (Table 1). Results of post hoc test showed a significant difference between the control group and group III ( $\mathrm{p}$ value 0.001 ), between group I and group III ( $\mathrm{p}$ value 0.001 ), between group II and group III ( $p$ value 0.001 ).

\section{Discussions}

This study discovered that there was significant difference in sperm quality

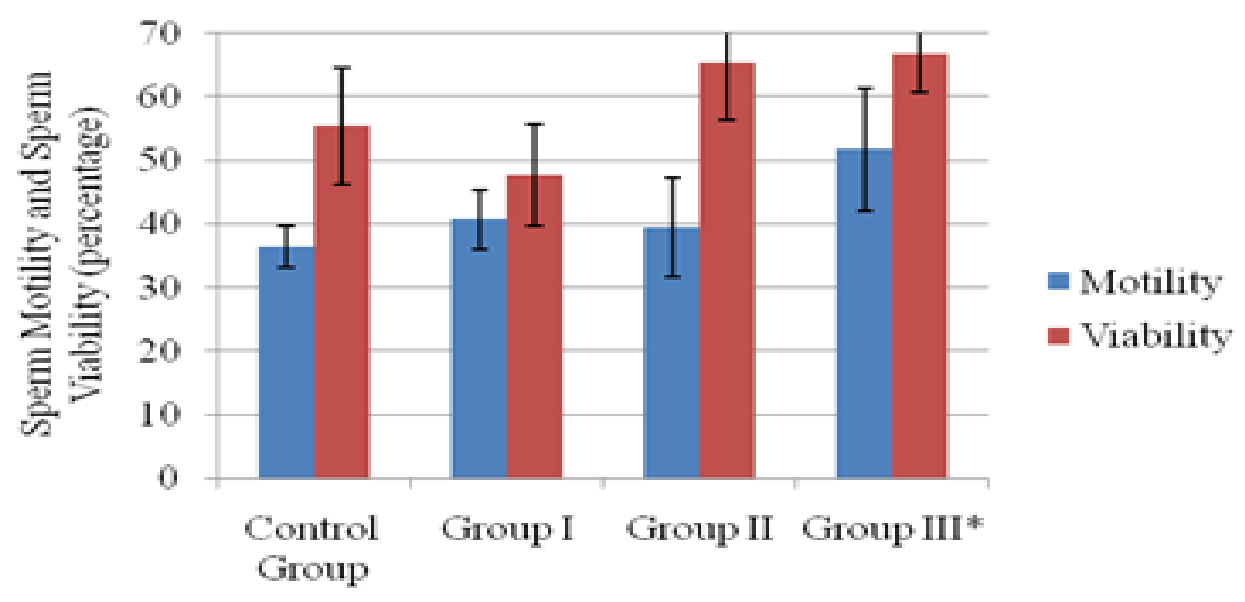

Figure 1 Mean Data of Sperm Motility Percentage and Sperm Viability Percentage

Note: * there was significance difference compared to control group. p-value $<0.05$ Error bar indicate standard deviation 


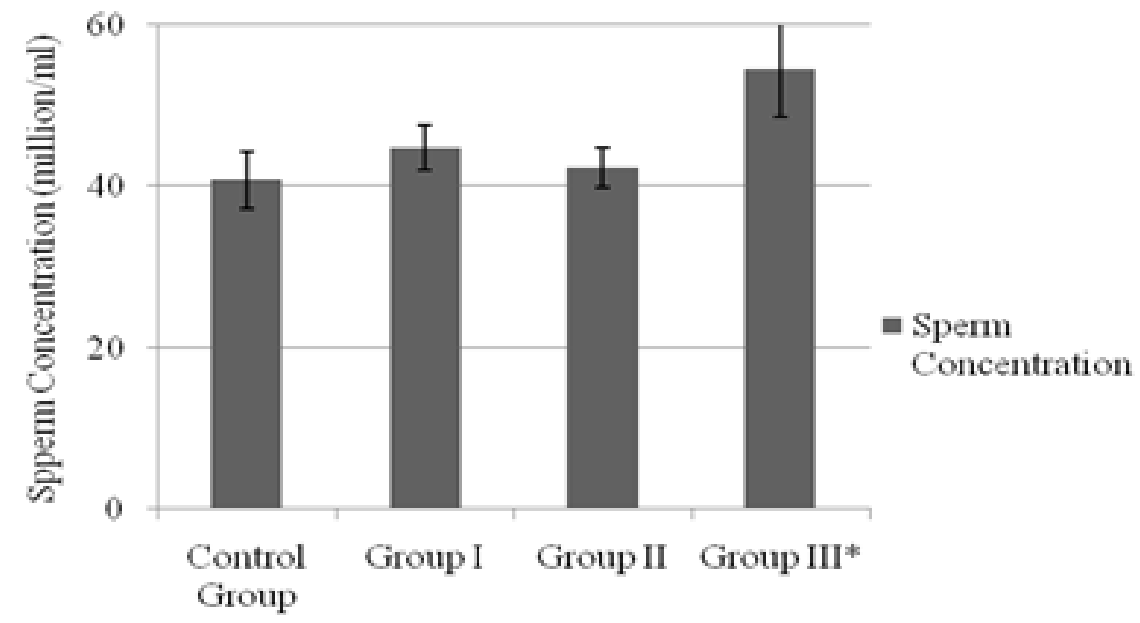

\section{Figure 2 Mean Data of Sperm Concentration}

Note: * there was significance difference compared to control group. p-value $<0.05$ Error bar indicate standard deviation

between the group III (suspension of $120 \mathrm{mg} /$ kg sappan wood ethanol extract) and control group. It is proven that the ethanol extract of the sappan wood with a dose of $120 \mathrm{mg} / \mathrm{kg}$ body weight had the effect of improving the quality of sperm including the percentage of progressively moveable sperm, the percentage of viable sperm and sperm concentration.

Sappan wood (Caesalpinia sappan. L) contains phenolic compounds which have four main components: (1) brazilin, (2) chalcone, (3) protosappanin, and (4) homoisoflavonoid. Brazilin, the main compound in the Sappan wood, has a variety of biological effects among them as antioxidant, antibacterial, anti-inflammatory, anti-hepatotoxity, and antiplatelet activity. ${ }^{6,10}$ As an antioxidant, brazilin may have an effect on spermatogenesis. Brazilin inhibits the production of nitrite oxide (NO) which acts as a mediator in the inflammatory reaction. Nitrite oxide produced by inducible nitrite oxide synthase (iNOS) may react with reactive oxygen species (ROS) and produces Nitrate Species that can cause DNA damage and gene mutations. In addition, the structure of the hydroxyl groups contained in brazilin which is a phenol plays a role as a major determinant in the free radical scavenging activity. The number and position of hydroxyl groups in carboxyl functional groups determines the antioxidant activity of brazilin. ${ }^{11,12,13}$

Reactive oxygen species is included in the oxidation process. The oxidation refers to a series of enzymatic reactions that normally occurs in the extracellular and intracellular signaling mechanisms. ${ }^{14}$ The main source of ROS derived from cell activities that require more oxygen, namely the mechanism of electron transport in the mitochondria. Regulation of ROS production and oxidationreduction reactions play an important role

Table 1 Mean Data of Sperm Motility Percentage, Sperm Viability Percentage and Sperm Concentration

\begin{tabular}{lccccc}
\hline \multirow{2}{*}{ Parameter } & \multicolumn{4}{c}{ Treatment Group } & \multirow{2}{*}{ P value } \\
\cline { 2 - 5 } & Control Group & Group I & Group II & Group III & \\
\hline Motility (\%,SD) & $36.39(3.28)$ & $40.67(4.69)^{* *}$ & $39.43(7.86)^{* *}$ & $51.75(9.63)^{*}$ & 0.005 \\
Viability (\%,SD) & $55.36(9.28)$ & $47.69(7.95)^{* *}$ & $65.44(9.15) \#$ & $66.63(6.07)^{*}$ & 0.002 \\
Concentration (million/ & $40.79(3.59)^{* *}$ & $44.85(2.80)^{* *}$ & $42.30(2.43)^{* *}$ & $54.47(5.81)^{*}$ & 0.001 \\
ml,SD) & & & & &
\end{tabular}

Note: The data are expressed as the mean \pm SD; ${ }^{*}$ significantly different with the control group; ${ }^{* *}$ significantly different with group III; \#significantly different with group I 
in normal spermatogenesis and sperm production. ${ }^{7}$

In semen, there are two main sources of ROS, leukositospermia and sperm. Most of the cemen contains leukocytes which were dominated by neutrophils. Reactive oxygen species is a byproduct of neutrophils-destroypathogenic mechanisms. ${ }^{15}$ Several preliminary studies showed a correlation between a decrease in sperm function with high levels of ROS and pro inflammatory mediators (IL-6, IL-8, and TNF- $\alpha$ ). ${ }^{7,14,15}$ Increased inflammatory mediators may cause injury to the sperm and the occurrence of lipid peroxidation in cell membranes of sperm. Besides leukocytes, sperm can also generate ROS. ${ }^{7}$

The ability of the sperm produces ROS related to the level of sperm maturation. In the process of sperm maturation, cytoplasmic residues normally removed before the sperm is released from the germinal epithelium. If there is a disruption in the maturation of sperm, there will be a build up of residual cytoplasm. Cytoplasmic residues are rich of Glucose-6phosphatase dehydorgenase that will produce nicotinamide adenine dinucleotide phosphate (NADPH). Nicotinamide adenine dinucleotide phosphate will generate ROS through the action of the enzyme NADPH oxidase. Increased ROS will eventually causes damage to sperm cells. ${ }^{7,14}$ Reactive oxygen species which derived from leukocytes known as external ROS, whereas ROS generated from the sperm itself is referred to internal ROS. The composition of the external and internal ROS affects the quality of sperm, both in DNA fragmentation, sperm motility, sperm concentration, and sperm morphology. Increased external ROS production associated with decreased sperm motility, sperm concentration and sperm morphology. The increase in internal ROS production associated with increased DNA fragmentation. ${ }^{7}$

The imbalance between the production of ROS as pro-oxidants and antioxidants can cause oxidative damage to cells which eventually led to the destruction of the quality of sperm. Brazilin as an antioxidant thought to have the ability to improve sperm quality by preventing oxidative stress that can be caused by exposure to the environment or produced by the sperm cell itself. 7,15

This result showed that that group I and group II, which were given sappan wood ethanol extract at a dose of $30 \mathrm{mg} / \mathrm{kg}$ bw and $60 \mathrm{mg} / \mathrm{kg} \mathrm{bw}$, respectively, showed results of sperm concentration and sperm motility percentage which was more than the control group. However, the difference between these groups was not significant, reveals that the ethanol extract of the sappan wood with dose of $30 \mathrm{mg} / \mathrm{kg}$ bw and $60 \mathrm{mg} / \mathrm{kg}$ bw had no effect on sperm quality of male rats. The optimum dose that affect to the improving sperm quality based on sperm motility percentage, sperm viability percentage, and sperm count of male wistar rat is $120 \mathrm{mg} / \mathrm{kg}$ bw of ethanol extracts of sappan wood.

This study had limitation. It was suspected that the parameter of sperm quality measurement could create a bias. In can be concluded that ethanol extract of sappan wood with a dose of $120 \mathrm{mg} / \mathrm{kg}$ for 35 days is able to improve sperm motility percentage, sperm viability percentage, and sperm concentration.

\section{References}

1. Jungwirth A, Giwercman A, Tournaye $H$, Diemer T, Kopa Z, Dohle G, et al. European Association of urology guidelines on male infertility: the 2012 update. Eur Urol. 2012;62(2):324-32.

2. Mascarenhas MN, Flaxman SR, Boerma T, Vanderpoel S, Stevens GA. National, regional, and global trends in infertility prevalence since 1990: a systematic analysis of 277 health surveys. PLos Med. 2012;9(12):e1001356.

3. Hasanpoor-Azghdy SB, Simbar M, Vedadhir A. The emotional-psychological consequences of infertility among infertile women seeking treatment : a result of qualitative study. Iran J Reprod Med. 2014;12(2):131-8.

4. Kim SJ, Kim MR, Hwang SY, Bae WJ, Kim $\mathrm{S}$, Hong $\mathrm{SH}$, et al. Preliminary report on the safety of a new herbal formula and its effect on sperm quality. World J Mens Health. 2013;31(3):254-61.

5. Imamovic Kumalic S, Pinter B. Review of Clinical trials on effects of oral antioxidants on basic semen and other parameters in idiopathic oligoasthenoteratozoospermia. Biomed Res Int. 2014;21(2):190-5.

6. Nirmal NP, Rajput MS, Prasad RG, Ahmad M. Brazilin from Caesalpinia sappan heartwood and its pharmacological activities: a review. Asian Pac J Tropmed. 2015;8(6):421-30.

7. Fujii J, Tsunoda S. Redox regulation of fertilisation and the spermatogenic process. Asian J Androl. 2011;13(3):4203.

8. Kementrian Kesehatan Republik Indonesia. Suplemen I farmakope herbal 
Indonesia. Jakarta: Kementrian Kesehatan Republik Indonesia; 2010.

9. WHO. WHO laboratory manual for the examination and processing of human semen [Internet]. 2010 [cited 12 Mei 2015]. Available from : http://www.who. int/reproductivehealth/publications / infertility/9789241547789/

10. Min BS, Cuong TD, Hung TM, Min BK, Shin BS, Woo MH. Compounds from the heartwood of Caesalpinia sappan and their anti-inflammatory activity. Bioorg Med Chem Lett. 2012;22(24):7436-9.

11. Bae I-K, Min H-Y, Han A-R, Seo E-K, Lee SK. Suppression of lipopoly saccharideinduced expression of inducible nitric oxide synthase by brazilin in RAW 264.7 macrophage cells. Eur J Pharmacol. 2005;513(3):237- 42.

12. Sasaki Y, Hosokawa T, Nagai M, Nagumo S. In vitro study for inhibition of NO production about constituents of Sappan Lignum. Biol Pharm Bull. 2007;30(1):193-6.

13. Balasundram N, Sundram K, Samman S. Phenolic compounds in plants and agri-industrial by-products: Antioxidant activity, occurrence, and potential uses. Food Chem. 2006;99(1):191-203 .

14. Tremellen K. Oxidative stress and male infertility--a clinical perspective. Hum Reprod Update. 2008;14(3):243-58.

15. Kefer JC, Agarwal A, Sabanegh E. Role of antioxidants in the treatment of male infertility. Int J Urol. 2009;16(5):449-57 